\title{
An Anti-Phishing mechanism for Single Sign-On based on QR-Code
}

\author{
Syamantak Mukhopadhyay \\ School of Electronics and Computer Science \\ University of Southampton \\ Southampton, UK \\ sm19g10@ecs.soton.ac.uk
}

\author{
David Argles \\ School of Electronics and Computer Science \\ University of Southampton \\ Southampton, UK \\ da@ecs.soton.ac.uk
}

\begin{abstract}
Today internet users use a single identity to access multiple services. With single sign-on (SSO), users don't have to remember separate username/password for each service provider, which helps the user to browse through the web seamlessly. SSO is however susceptible to phishing attacks. This paper describes a new anti phishing SSO model based on mobile QR code. Apart from preventing phishing attacks this new model is also safe against man in the middle \& reply attacks.
\end{abstract}

Keywords-authentication; single sign-on; phishing; onetime password; QR code

\section{INTRODUCTION}

Internet is becoming more and more user centric each day. With the advent of web 2.0 internet users are becoming more inclined to use services from multiple content and service providers (CSP or SP). Most SPs provide user registration service whereby a user can create his/her own account and maintain it. As such a user has to maintain separate user accounts (username and password) for each of the SPs he/she uses. A study shows that today a typical user needs to maintain about twenty five different accounts which require password and uses eight of them in a given day[1]. Not only is this approach annoying to the user, it also raises some serious security questions, e.g. password fatigue [1].

Single sign-on(SSO) is one approach which aims to address the root cause of this problem[2]. With single sign-on, a user can create one account and use that account to login once and use multiple services hosted in different domains. There are three components or actors of a single sign-on system. A IdP or "identity Provider", a "Service Provider" (SP) or "Relying Party" (RP) and the user. RP relies on $\operatorname{IdP}(s)$ to authenticate user credentials. SSO approach outsources the responsibility of user authentication from service providers to IdPs. Not only does SSO reduces the burden of the user, a SSO system can also enable users to share contents between different service providers[3]. Many commercial solutions exist which provide SSO service, such as OpenID[4], Information Card[5] and SAML [6] based SSO Shibboleth.

In the next section we will go through these models and see their limitations.

\section{SingLE Sign-ON PROCESS}

\section{A. Existing Systems}

Shibboleth : Shibboleth is an open source single sign-on solution which is best suited for portal or Intranet applications[7]. It uses Security Assertion Markup Language (SAML), an OASIS specification for $\mathrm{xml}$ based security assertions. There are two main components of shibboleth system namely "Identity Provider" (IdP) and "Service Provider" (Sp).

In this system if a user wants to access a service or a resource of a SP, the SP redirects the user's web browser to a WAYF server. WAYF server displays a set of organizations to the user from which the user chooses one. Once the user chooses an organization, user's browser is redirected to corresponding login page of IdP and user provides his login credentials. Once the user successfully logs in, user's browser is redirected back to SP who decides whether to enable access for this user or not.

OpenID: OpenID[4] provides a user centric authentication model in a sense user can chose to implement his/her own OpenID provider or selects from a list of existing OpenID providers. Main components of an OpenID system are UserAgent, Relying Party (RP) and OpenID Provider (IdP).

User initiates the authentication process with RP using "User Agent". RP then, depending of user provided identifier, discovers user's IdP and redirects "User Agent" to IdP with an authentication request. User performs authentication at IdP side with username/password and IdP then redirects "User Agent" to RP again with a security assertion message specifying whether authentication has succeeded or failed. Based on this assertion RP decides whether to grant the user permission to access its services or not.

Major advantage of OpenID is it doesn't require any preestablished contract between RPs and IdPs. But it is susceptible to phishing attack[8].

Information Card: Information Card is based on real world multi identity concept[9]. Like Driving license, passport etc an Information Card user can have different identity sectors. Each 
sector contains a different assertion which can be provided by different identity providers. When a user accesses services from a RP he/she logins in with one of the identity sectors or cards instead of username/password, with rest of the identity sectors remains hidden from RP.

Microsoft Cardspace is a SSO solution from Microsoft based on information card approach[10]. Information card approach provides more flexibility than username/password approach. As the information cards or sectors can be encrypted, it is also more secure than simple username/password. But with respect to OpenID, it is a very heavy system. Different Information Card identity sectors are stored in user computer which makes it susceptible to various security/privacy issues[8].

\section{B. Phishing Vulnerability in SSO}

Whoever there are certain security limitations of SSO systems. SSO involves crossing security domains between different SPs. Moreover most IdPs rely on username/password as their preferred authentication method. And as such it is susceptible to phishing attacks[11] [12] [13].

From the above discussion, login procedure of most of the Single Sign-On systems can be generalized to a set of common steps[14]. At the first phase user establishes unique identity by registering to an IdP. At the second phase user accesses services provided by different RPs by the following steps:

1. User requests a Service Provider or Relaying Party (say RP1) to access services provided by the RP1. RP1 initiates the SSO process.

2. RP1 redirects the request to Identity Provider (IdP1) in order to authenticate the user.

3. User authentication is done by username/password method or certificates.

4. IdP1 asserts user credentials to RP1 by sending an application ticket (AuthToken1) or assertion.

5. Based on the assertions RP decides whether to provide service to the user or not.

6. If the user wants to access services from another service provider RP2, RP1 will forward AuthToken1 to RP2. RP2 then can verify user credentials with IdP1 just by sending AuthToken1 to IdP1. User doesn't need to provide his/her credentials again.

Fugure 1. provides the pictorial representation of the steps.

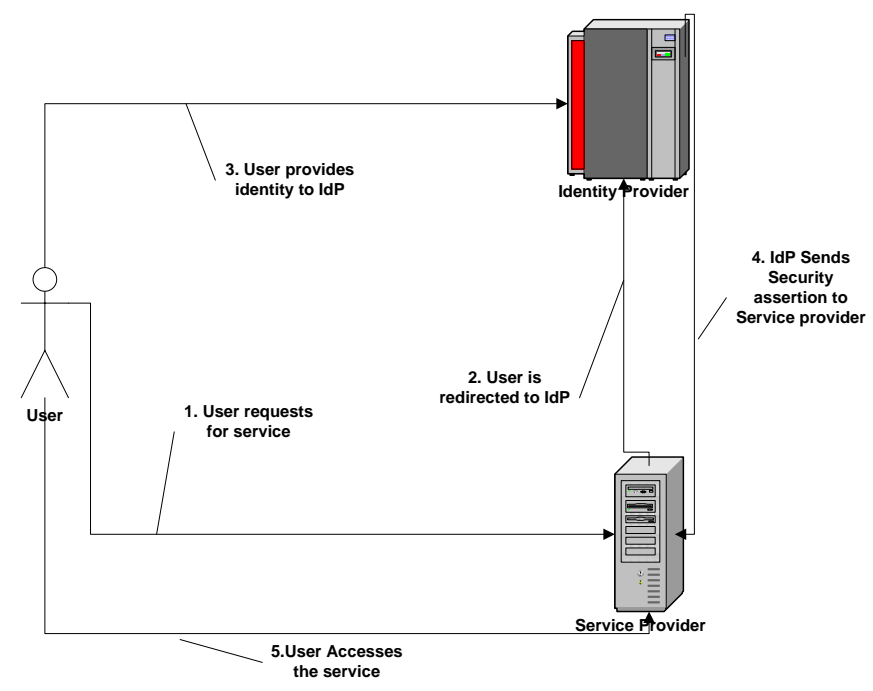

Figure 1. SSO general model

It is at stage $2 \& 3$ that the system is susceptible to phishing. Malicious RP can display a phishing page and as the user enters his/her username and password, RP can obtain details about user credentials. Since there is no way for the user to authenticate RP, he/she becomes vulnerable to phishing attack[15].

As can be seen most of the SSO systems are susceptible to "verifier impersonation" or phishing attacks as they use password for credential verification[16]. Phishing is a major issue in today's internet oriented life which causes massive financial loss every year[17]. It is therefore important to prevent phishing in SSO.

In the next section we will see previous works that have been done to prevent phishing in SSO systems.

\section{PREVIOUS WORKS NO ANTI PHISHING MECHANISM FOR SSO}

Various client side solutions exist which can detect a phishing page. E.g. Personal icon from myOpenID which can be used on a particular user's PC only. Solutions are provided by Verising (Validation Certificate for IE7 and seatbelt for Firefox) but they are browser dependent and not cost effective.

An improved SSO solution has been proposed by [18] based on Kerberos[19]. This model uses two passwords instead of one, one by authentication server and one by ticket granting server. Although this model adds one extra level of security, it is of little help to prevent phishing in a distributed web applications. Phishing web pages can be created to simulate this two phase approach and obtain both the passwords.

Another approach is to use mobile SIM in authentication phase of a $\mathrm{SSO}[20]$. As proposed in this model, during login phase SIM is authenticated and proof of authentication is presented to the identity provider (IdP). IdP then lets the user login successfully. One drawback of this approach is that the authentication is carried out at the client side. 
Lee \& Jeun proposed a new approach to address the issue of phishing in SSO[15]. Every time user wants to access the service from a RP, a new token will be generated and will be sent to the user's email address. User then can login with the token. Although it solves the phishing problem, this solution breaks the basic philosophy of SSO. User needs to sign on to his/her email first to access the generated token. It can be thought of a SISO (single identity sign on) as it requires users to sign in twice.

You \& Jun proposed a solution to phishing problem in SSO by using I-PIN[21]. But this solution can't be implemented globally.

We believe one possible solution to phishing problem in SSO is to use a onetime password approach. In the next section we will propose our solution for SSO with onetime password scheme.

\section{PROPOSED SSO MODEL WITH ONETIME PASSWORD}

Apart from addressing the issue of phishing in SSO, the new model should also be simple enough, so that it can be adopted in a real life scenario. In other words the proposed model should not introduce any new steps or complexity into the SSO process for the users. We will select our onetime password generation schema with these goals in mind.

PKI based onetime password generation model described by [22] provides good security features. But this model requires several additional steps during user registration and authentication phases.

For our proposed model we will make use of QR-code based onetime password schema[23]. Our idea is based on the assumption that most of the internet users today are equipped with a mobile phone that has a camera.

The proposed model has three entities: an Identity Provider $(\mathrm{IdP})$, a Service Provider (SP) and a User $\left(\mathrm{U}_{\mathrm{A}}\right)$. The model is divided in two phases: User Registration and User Verification.

TABLE I. NOTATIONS

\begin{tabular}{|l|l|}
\hline \multicolumn{1}{|c|}{ Notation } & \multicolumn{1}{c|}{ Meaning } \\
\hline $\mathrm{ID}_{\mathrm{A}}$ & Username or identity of the User \\
\hline $\mathrm{RP}_{\mathrm{A}}$ & Root password of the user \\
\hline $\mathrm{X}_{\mathrm{A}}$ & Secret key of the user \\
\hline $\mathrm{E}_{\mathrm{QR}}$ & Encoded QR code \\
\hline $\mathrm{D}_{\mathrm{QR}}$ & Decoded $\mathrm{QR}$ code \\
\hline
\end{tabular}

\section{A. User Registration Phase}

1. During this phase $\mathrm{U}_{\mathrm{A}}$ provides his/her $\mathrm{ID}_{\mathrm{A}}$ and $\mathrm{RP}_{\mathrm{A}}$ to IdP.

2. Based on $\mathrm{RP}_{\mathrm{A}}$, IdP calculates a key $\mathrm{X}_{\mathrm{A}}$ using a one way hash function.

3. IdP sends $X_{A}$ to user's mobile device and it gets stored as a secret key.
Fugure 2. below provides the pictorial representation of the steps.

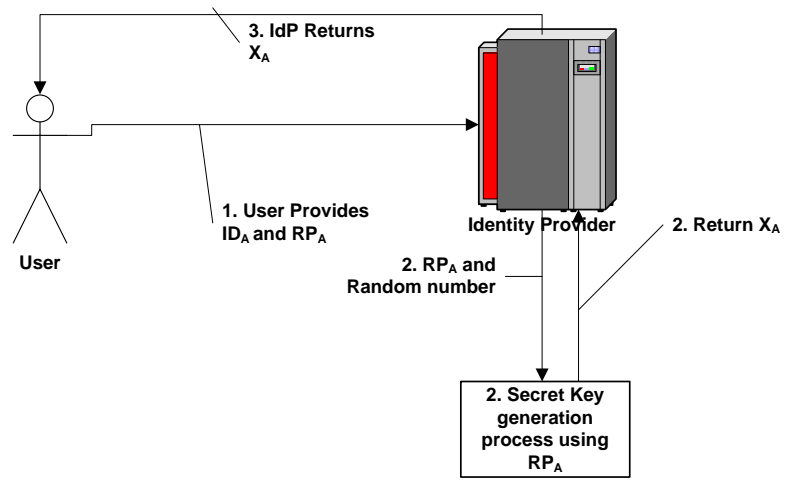

Figure 2. User Registration Phase

\section{B. User Verification Phase}

1. User wants to access a service provided by SP.

2. SP redirects user to its IdP.

3. User provides his/her identity to IdP.

4. IdP then based on user's identity calculates $\mathrm{X}_{\mathrm{A}}$. IdP uses $\mathrm{X}_{\mathrm{A}}$ and a random number to calculate the $\mathrm{QR}$ code $\mathrm{E}_{\mathrm{QR}}$.

5. IdP then sends $\mathrm{E}_{\mathrm{QR}}$ and a timestamp $\mathrm{T} 1$ to the user.

6. User uses the embedded camera in his/her mobile device and stored $\mathrm{X}_{\mathrm{A}}$ to decode the $\mathrm{QR}$ code to $\mathrm{D}_{\mathrm{QR}}$. $\mathrm{D}_{\mathrm{QR}}$ and timestamp $\mathrm{T} 2$ are then sent to the IdP.

7. IdP checks the validity of $\mathrm{D}_{\mathrm{QR}}$ and acceptability of $\mathrm{T} 2$ and based on that sends a security assertion to SP.

8. SP then, based on the security assertion can allow or deny use of its services.

9. If the user now wants to access services from another service provider (SP1), SP can forward the assertion token to SP1 maintaining the basic principles of SSO.

Fugure 3. below provides the pictorial representation of the steps.

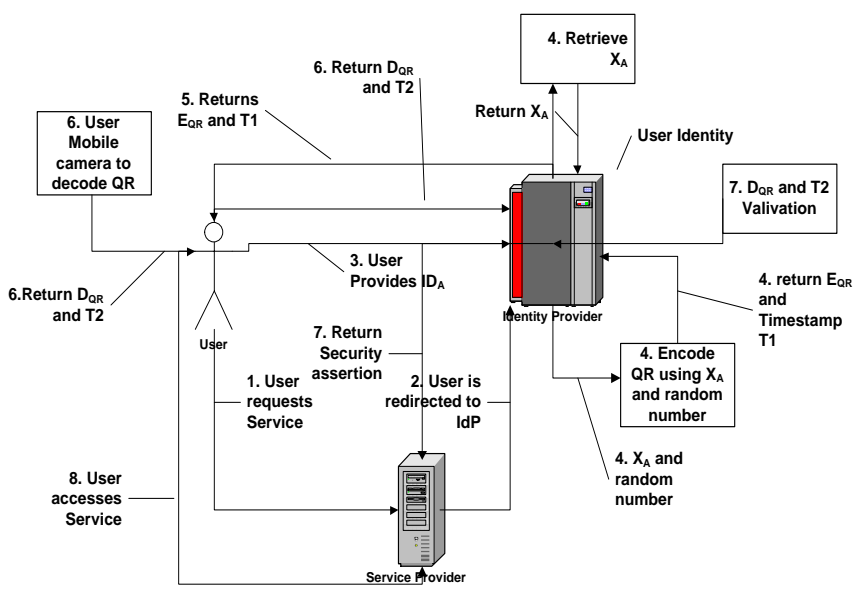

Figure 3. User Verification Phase 


\section{User Interaction}

From the user perspective this new model is quiet simple to use. When the user is redirected to Identity Provider's login page, he/she needs to provide the identity (username) only.
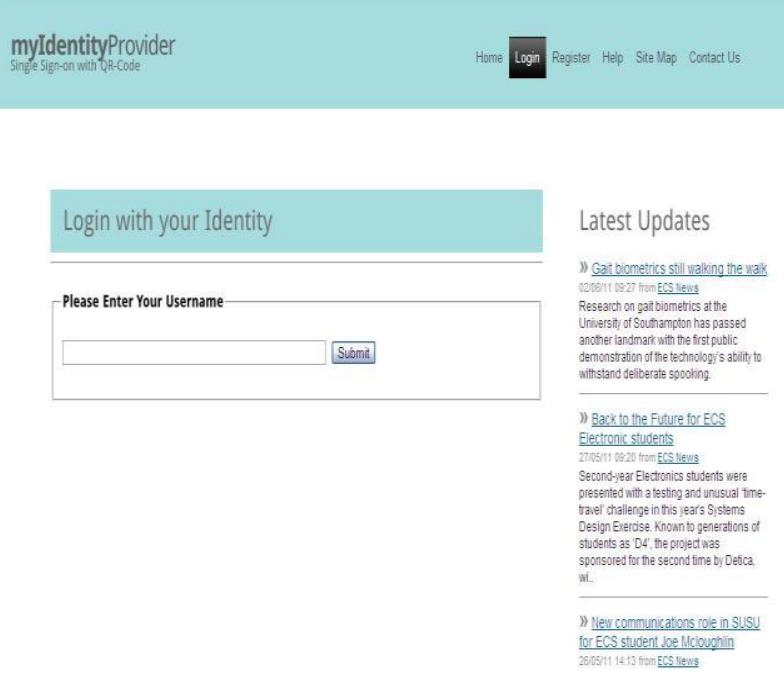

Figure 4. User Login step 1

Upon receiving the identity of the user, IdP generates the QR-Code using the mechanism described before.
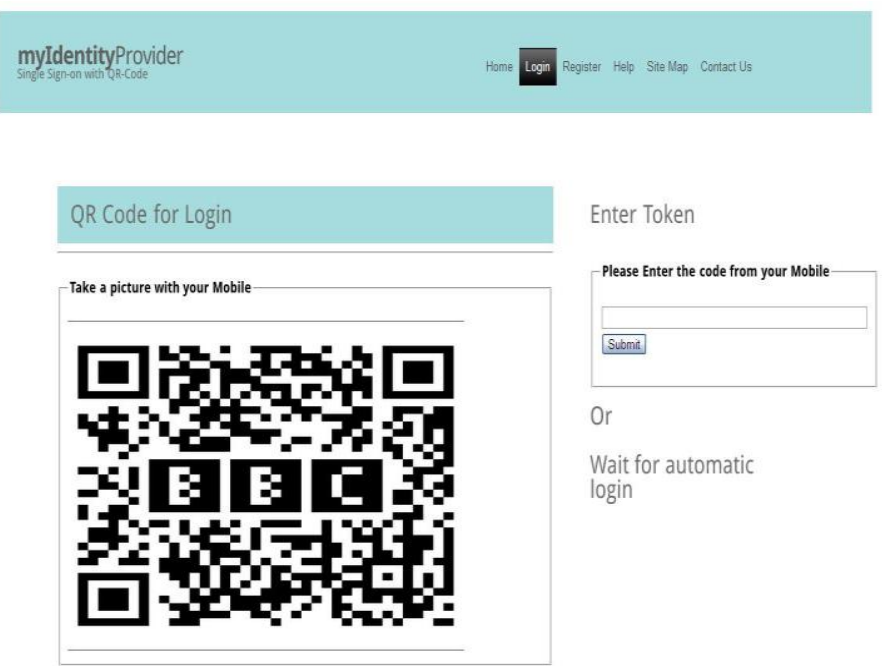

Figure 5. User Login step 2

The user then uses his/her mobile device to take a picture of the QR-Code. An app in user mobile device uses the secret key $\mathrm{X}_{\mathrm{A}}$ already stored in it (during registration phase) to decode the QR-Code. If the user's mobile device is web enabled, the app can directly send the decoded value and timestamp to the IdP using HTTPS. Alternatively this decoded value will be displayed to the user who can then enter the value manually to login. In either case, this schema doesn't introduce any new complications for users.

Generation of the secret key $X_{A}$ should be dynamic. i.e if the user's $\mathrm{X}_{\mathrm{A}}$ is compromised due to loss of mobile device or any other reason, he/she will be able to generate a new secret key. Since $X_{A}$ is generated from $\mathrm{RP}_{\mathrm{A}}$, All the user needs to do is to reset his/her $\mathrm{RP}_{\mathrm{A}}$.

\section{SECURITY ANALYSIS}

\section{A. Phishing attack}

Since the root password $\mathrm{RP}_{\mathrm{A}}$ is never disclosed during the verification phase, this model is fairly resistive to phishing attacks. Further if the secret key $\mathrm{X}_{\mathrm{A}}$ is compromised at any stage, $\mathrm{U}_{\mathrm{A}}$ can change it by simply resetting $\mathrm{RP}_{\mathrm{A}}$ at the IdP side. Since $X_{A}$ is generated using one way hash function, it is unfeasible to derive $\mathrm{RP}_{\mathrm{A}}$ from $\mathrm{X}_{\mathrm{A}}$.

\section{B. Other attacks}

Assuming timestamp difference (T1-T2) acceptable by the IdP is minimum, If a man in the middle intercepts $\mathrm{D}_{\mathrm{QR}}$ and tries to emulate the user, timestamp of $\mathrm{D}_{\mathrm{QR}}$ would be expired. In addition, as $\mathrm{E}_{\mathrm{QR}}$ is generated using a random number, after the allowed time interval IdP will select a new random number. Hence this model is fairly safe from both man in the middle attacks and reply attacks[23].

\section{CONCLUSION}

In this paper we went through a brief overview of SSO process and analyzed its vulnerability against phishing attacks. We then presented a new SSO model with mobile QR code based onetime password schema. Security analysis of our model shows that apart from preventing phishing attacks, our model is safe against man in the middle \& reply attacks as well. This model is simple from usability perspective and since most users today are equipped with camera embedded mobile device, this model can be adopted universally.

\section{REFERENCES}

[1]
D. Florencio and C. Herley, "A large-scale study of web password habits," in 16th International World Wide Web Conference, WWW2007, May 8, 2007 - May 12, 2007, Banff, AB, Canada, 2007, pp. 657-666.

R. Dhamija and L. Dusseault, "The Seven Flaws of Identity Management: Usability and Security Challenges," Security \& Privacy, IEEE, vol. 6, pp. 24-29, 2008.

S. San-Tsai, et al., "Towards Enabling Web 2.0 Content Sharing beyond Walled Gardens," in Computational Science and Engineering, 2009. CSE '09. International Conference on, 2009, pp. 979-984.

OpenID, "OpenID Authentication 2.0," December 5, 20072007.
A. Nanda and M. B. Jones, "Identity Selector Interoperability Profile," July 20082008.

Oasis, "Security Assertion Markup Language (SAML) V2.0," 15 March 20052005.

"Internet2. Shibboleth System," http://shibboleth.internet2.edu/. S.-T. Sun, et al., "OpenIDemail enabled browser: towards fixing the broken web single sign-on triangle," presented at the Proceedings of the 6th ACM workshop on Digital identity management, Chicago, Illinois, USA, 2010. 
[9] "Identity Metasystem Interoperability Version 1.0 Committee Draft," http://informationcard.net/specifications, 10 November 20082008.

[10]

"Microsoft

Cardspace,"

http://www.microsoft.com/windows/products/winfamily/cardspace/ default.mspx.

[11] E. Maler and D. Reed, "The Venn of Identity: Options and Issues in Federated Identity Management," Security \& Privacy, IEEE, vol. 6, pp. 16-23, 2008.

[12] W. D. Yu, et al., "A phishing vulnerability analysis of web based systems," in Computers and Communications, 2008. ISCC 2008. IEEE Symposium on, 2008, pp. 326-331.

[13] Z. A. Khattak, et al., "A study on threat model for federated identities in federated identity management system," in Information Technology (ITSim), 2010 International Symposium in, 2010, pp. 618-623.

[14] P. B. Tiwari and S. R. Joshi, "Single sign-on with one time password," in Internet, 2009. AH-ICI 2009. First Asian Himalayas International Conference on, 2009, pp. 1-4.

[15] L. HwanJin, et al., "A New Anti-phishing Method in OpenID," in Emerging Security Information, Systems and Technologies, 2008. SECURWARE '08. Second International Conference on, 2008, pp. 243-247.

[16] O. Hyun-Kyung and J. Seung-Hun, "The Security Limitations of SSO in OpenID," in Advanced Communication Technology, 2008. ICACT 2008. 10th International Conference on, 2008, pp. 16081611.
[17] D. Irani, et al., "Evolutionary study of phishing," in eCrime Researchers Summit, 2008, 2008, pp. 1-10.

[18] J. Yang, "An Improved Scheme of Single Sign-On Protocol Based on Dynamic Double Password," in Environmental Science and Information Application Technology, 2009. ESIAT 2009. International Conference on, 2009, pp. 572-574.

[19] B. C. Neuman and T. Ts'o, "Kerberos: an authentication service for computer networks," Communications Magazine, IEEE, vol. 32, pp. 33-38, 1994.

[20] I. Jrstad, et al., "Releasing the potential of OpenID \& SIM," in Intelligence in Next Generation Networks, 2009. ICIN 2009. 13th International Conference on, 2009, pp. 1-6.

[21] Y. Jae-Hwe and J. Moon-Seog, "A Mechanism to Prevent RP Phishing in OpenID System," in Computer and Information Science (ICIS), 2010 IEEE/ACIS 9th International Conference on, 2010, pp. 876-880.

[22] H. C. Kim, et al., "A Design of One-Time Password Mechanism Using Public Key Infrastructure," in Networked Computing and Advanced Information Management, 2008. NCM '08. Fourth International Conference on, 2008, pp. 18-24.

[23] L. Kuan-Chieh, et al., "A One-Time Password Scheme with QRCode Based on Mobile Phone," in INC, IMS and IDC, 2009. NCM '09. Fifth International Joint Conference on, 2009, pp. 2069-2071. 